\title{
Beta Reactivity, Prospective Facilitation of Executive Processing, and Its Dependence on Dopaminergic Therapy in Parkinson's Disease
}

\author{
Ashwini Oswal, ${ }^{1,2 *}$ Vladimir Litvak, ${ }^{2 *}$ Paul Sauleau, ${ }^{3}$ and Peter Brown ${ }^{1}$ \\ ${ }^{1}$ Department of Clinical Neurology, John Radcliffe Hospital, Oxford OX3 9DU, United Kingdom, ${ }^{2}$ Wellcome Trust Centre for Neuroimaging, UCL Institute \\ of Neurology, London WC1N 3BG, United Kingdom, and ${ }^{3}$ Behavior and Basal Ganglia Unit, University of Rennes, 35033 Rennes, France
}

Oscillatory activity in the beta frequency band has been shown to be modulated during the preparation and execution of voluntary movements at both cortical and subcortical levels. The exaggeration of beta activity in the basal ganglia of patients with Parkinson's disease has heightened interest in this phenomenon. However, the precise function, if any, subserved by modulations in beta activity remains unclear. Here we test the hypothesis that beta reactivity can be dissociated from processing of specific actions and can index the salience of cues with respect to future behavior in a way that might help prospectively prioritize resources. To this end we used an experimental paradigm designed to dissociate salient warning cues from processing of specific motor or cognitive actions. We recorded local field potential activity from the subthalamic nucleus of humans undergoing functional neurosurgery for the treatment of Parkinson's disease, while the same patients were on or off the dopamine prodrug levodopa. In this way we demonstrate that beta reactivity is indeed dependent on the salience of cues with respect to future motor and cognitive action and is promoted by dopamine. The loss of normal beta encoding of saliency may underlie some of the motor and cognitive features of basal ganglia disorders such as Parkinson's disease.

\section{Introduction}

Synchronized neural oscillations at beta frequencies have been reported in the basal ganglia of patients with Parkinson's disease (PD) and models of this disease (Hammond et al., 2007). Their tonic and phasic suppression by dopaminergic medication and salient cues correlates with changes in motor performance (Kühn et al., 2004, 2009; Doyle et al., 2005; Williams et al., 2005; Kempf et al., 2007; Ray et al., 2008; Pogosyan et al., 2009; López-Azcárate et al., 2010; Zaidel et al., 2010). Such suppression is accompanied by a surge in dynamic interactions between basal ganglia neurons, involving increased neuronal discharge rates and synchronization at gamma, or higher, frequencies (Courtemanche et al., 2003; Foffani et al., 2003; Amirnovin et al., 2004; Alegre et al., 2005; Devos et al., 2006; Pogosyan et al., 2006; Kempf et al., 2007; Androulidakis et al., 2008; Kane et al., 2009; López-Azcárate et al., 2010; Özkurt et al., 2011). This raises two possibilities. First, beta

\footnotetext{
Received Jan. 18, 2012; revised April 16, 2012; accepted May 22, 2012.

Author contributions: P.S. and P.B. designed research; P.S. and P.B. performed research; A.0. and V.L. analyzed data; A.O., V.L., and P.B. wrote the paper.

P.B. is funded by the Medical Research Council UK, Wellcome Trust, Rosetrees Trust, and the National Institute for Health Research (NIHR) Biomedical Research Centre, Oxford. A.0. is funded by the NIHR Academic Clinical Fellowship program at the University of Oxford. V.L. is funded by the Wellcome Trust. We are grateful to Alexandre Eusebio, Wesley Thevathasan, Alek Pogosyan, Ludvic Zrinzo, Keyoumars Ashkan, Tipu Aziz, Wim Vandenberghe. and Bart Nuttin for their help with data collection.

*A.O. and V.L. contributed equally.

The authors declare no competing financial interests.

Correspondence should be addressed to Professor Peter Brown, Department of Clinical Neurology, University of Oxford, John Raddliffe Hospital, 0X3 9DU, UK. E-mail: peter.brown@clneuro.ox.ac.uk.

DOI:10.1523/JNEUROSCI.0275-12.2012

Copyright $\odot 2012$ the authors $\quad 0270-6474 / 12 / 329909-08 \$ 15.00 / 0$
}

suppression could be an epiphenomenon of the shift in neuronal patterning that underscores movement. Second, beta suppression might be necessary to permit the dynamic reconfiguration of neural networks. The distinction is important as in one case beta suppression is an emergent epiphenomenon, inseparable from motor processing, whereas in the other it is an active process that prospectively sets the stage for rate coding and spectral interactions at higher frequencies during motor processing.

Although there is some support for the latter suggestion (Kühn et al., 2004; Pogosyan et al., 2009; Chen et al., 2011; Ray et al., 2012), beta suppression in the basal ganglia has never been disassociated from motor processing in experimental situations, and hence its role as an active and prospective control mechanism remains speculative (Jenkinson and Brown, 2011).

The above issue may also relate to another unsettled question. Dopaminergic medication is effective in suppressing tonic levels of beta synchrony in the basal ganglia (Hammond et al., 2007), but what is more contentious is whether it also improves the phasic reactivity of beta synchrony to salient internal and external cues (Doyle et al., 2005; Androulidakis et al., 2008) or not (Devos et al., 2006). Perhaps, the conflation of different phenomena has confounded such studies.

Finally, it has recently been stressed that the above is a restrictively motoric view of beta suppression, and that the latter may represent a generic control process in executive function, be it primarily motor or cognitive in its nature (Engel and Fries, 2010). These considerations lead to the hypothesis that beta suppression in the basal ganglia can be an active process involved in the prospective control of motor or cognitive readiness (Engel and Fries, 
2010; Jenkinson and Brown, 2011) and can thus be disassociated from specific motor parameterization and cognitive processing. Further, this prospective control function of beta synchrony is modulated by dopamine. Accordingly, we developed a paradigm that separates warning of the need for a response from the processing of the specific response, and tested this while recording beta activity from the subthalamic nucleus in patients with $\mathrm{PD}$ on and off the dopamine prodrug, levodopa.

\section{Materials and Methods}

Ten patients ( 8 male and 2 female) with idiopathic Parkinson's disease who underwent implantation of deep brain stimulation electrodes in the subthalamic nucleus (STN) at four different surgical centers participated in the study. Each was implanted for the treatment of their Parkinson's disease and with the exception of a single patient, all patients were implanted bilaterally. All patients gave informed written consent to participate in the study, which was approved by the local ethics committees of the different surgical centers. The clinical characteristics of the patients, surgical details, and electrode localization have been described in detail in a previous study (cases 1-3, 6, 8-12, and 14; Sauleau et al., 2009). Note that because we were particularly interested in determining any interaction between dopaminergic therapy and beta reactivity, we excluded four subjects from the original report by Sauleau et al. (2009): three with only on or off recordings and one in whom prominent rest tremor off medication led to contamination of local field potential (LFP) spectra by movement artifact at tremor frequencies and harmonics thereof.

Five patients were implanted at the University Hospital Gasthuisberg, Leuven, Belgium; three patients were implanted at the Oxford Radcliffe Hospital, Oxford, UK; one patient was implanted at King's College Hospital, London, UK; and one patient was implanted at the National Hospital for Neurology and Neurosurgery, London, UK. None of the patients had any surgical complications, postoperative confusion, or severe motor impairment that precluded understanding or performance of the test. Paradigm. We developed a paradigm that separated warning of the need for a response from the processing of the specific response. This was achieved by presenting a warning cue that signaled the need for a motor or cognitive response followed by an imperative cue which specified the precise response required. At the same time, temporal expectancy was limited by pseudo-randomization of the inter-cue interval. Our prediction was that salient warning cues would elicit beta suppression, even when the necessary response and its timing were obscure. Subjects were seated comfortably in a chair. The test consisted of three conditions: a 'standard motor' task and a 'control motor' task consisted of performing body movements according to visual cues presented on a laptop computer, while the remaining condition was a non-motor 'counting' task consisting of counting in silence identical cues while at rest. Each trial began with a fixation cross and then presentation of a warning cue. This could be a black circle ( $50 \%$ probability), square ( $25 \%$ probability), or triangle ( $25 \%$ probability), which substituted the fixation cross provided between trials to limit confounding eye movements (Fig. 1). The warning cue was followed by a schematic of the body (imperative cue) with a single segment highlighted in red (head, trunk, left arm, right arm, left leg or right leg, with equal probabilities). In the standard motor task, patients were instructed to move the highlighted body segment whenever preceded by a circle (target cue), but to ignore the body schema cue when this was preceded by either a non-target square or triangle (Fig. 1). This was reversed in the control motor task, in which patients were instructed to move the highlighted body segment whenever preceded by a square or triangle (target cue), but to ignore the body schema cue when a nontarget circle preceded this. In the non-motor counting task, the patients were asked to remain at rest and to count silently in their mind how many left arms were highlighted in red after circles (target cues), ignoring nontarget squares and triangles. At the end of the task the subjects were asked to report this number. The experiment began with some demonstration trials in which the intended movements were shown to the patient. These movements consisted of single and brief flexion of the neck, flexion of the trunk, left or right wrist extension, and left or right ankle dorsiflexion. Each of the three tasks was repeated twice, the six blocks being presented

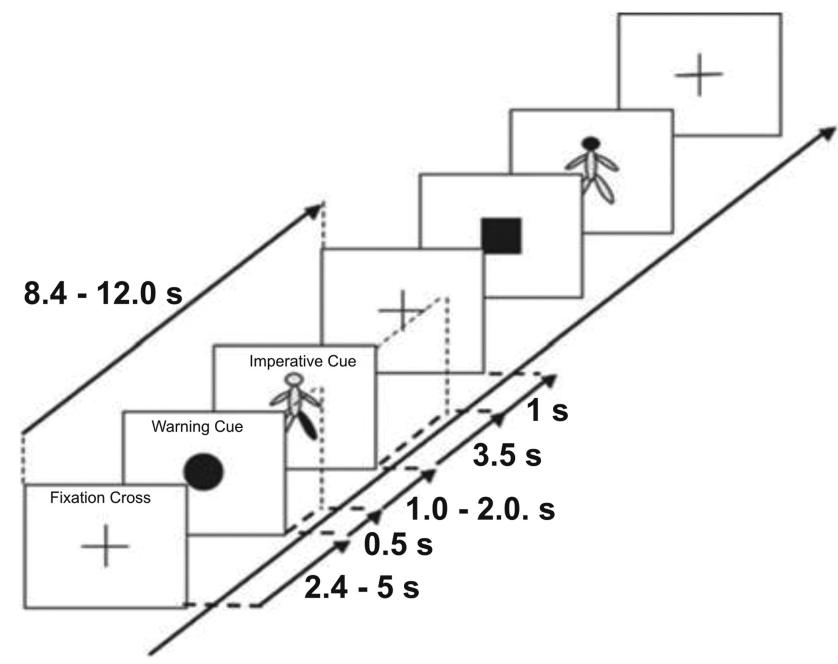

Figure 1. Schematic of two trials during the standard motor task. In this block type patients were instructed to move the highlighted body part indicated in the body schema only when the warning cue was a circle. They were instructed to remain at rest when the body schema was preceded by either a square or a triangle warning cue. The behavioral relevance of the stimuli was reversed in the control motor task. A similar sequence was also used in the counting task.

in a pseudo-randomized order across patients. The task requirements were verbally explained to the subject before the start of each block. Blocks of a given task lasted $4 \mathrm{~min}$, and were followed by 1-2 min rest. Each block of a given task consisted of 24 trials, giving a total number of 48 trials per condition. Each trial started with the presentation of a fixation cross at the center of a portable PC screen, followed 2.4-5.0 s later by the warning cue of fixed duration $(0.5 \mathrm{~s})$ and by a $3.5 \mathrm{~s}$ duration second cue of a body schema presented at the center of the screen. This was followed by a blank screen for $1 \mathrm{~s}$, before the next trial began with reappearance of the fixation cross. The total duration of the recordings was $\sim 3 \mathrm{~h}$, although an hour or so of this was spent resting while the effects of medication took hold.

Within each block, trials occurred pseudo-randomly, every 8.4-12.0 s. The two consecutive cues were presented with a randomized interval between cue onsets (foreperiod before imperative cue) of 1.5-2.5 s. This foreperiod includes the period of warning cue presentation which was of $0.5 \mathrm{~s}$ duration. Note that the foreperiod duration of $1.0-1.6 \mathrm{~s}$ given by Sauleau et al. (2009) is erroneous and should be identical to that given here. The distribution of the foreperiod in the present paradigm was uniform, meaning that the probability of the target cue appearing at any point in the interval was fixed. Moreover, in choosing similar overall probabilities of target and non-target cues, we biased the experimental design so that evoked activity related to behavioral relevance would be stressed over that related to novelty. Thus, our experimental design differed from previous $\mathrm{P} 300$ and contingent negativity variation paradigms insofar as the response to the warning cue was disambiguated from specific task-related motor or cognitive processing and behavioral relevance emphasized over novelty.

Patients were kept under constant visual inspection by the researcher and error trials noted. There were two types of error. The first related to the warning cue and involved patients subsequently moving when they should not have or failing to move when they should have. On average, patients made such errors in $1.2 \%$ of trials Off and On medication (total number of trials in each drug state was 960 when the two motor tasks were combined). The second type of error was related to the imperative cue, and involved patients moving the wrong side or body segment. On average, patients made such errors in $1.0 \%$ of trials Off and On medication (total number of trials in each drug state was 960 when the two motor tasks were combined). Because we were primarily interested in the evoked response to the warning cue, we removed those trials with the first type of error as attention to the warning cue could not be ensured in these instances. This left $44-48$ of 48 trials in each subject per motor task in each drug state without errors. In the counting task, four patients 
A

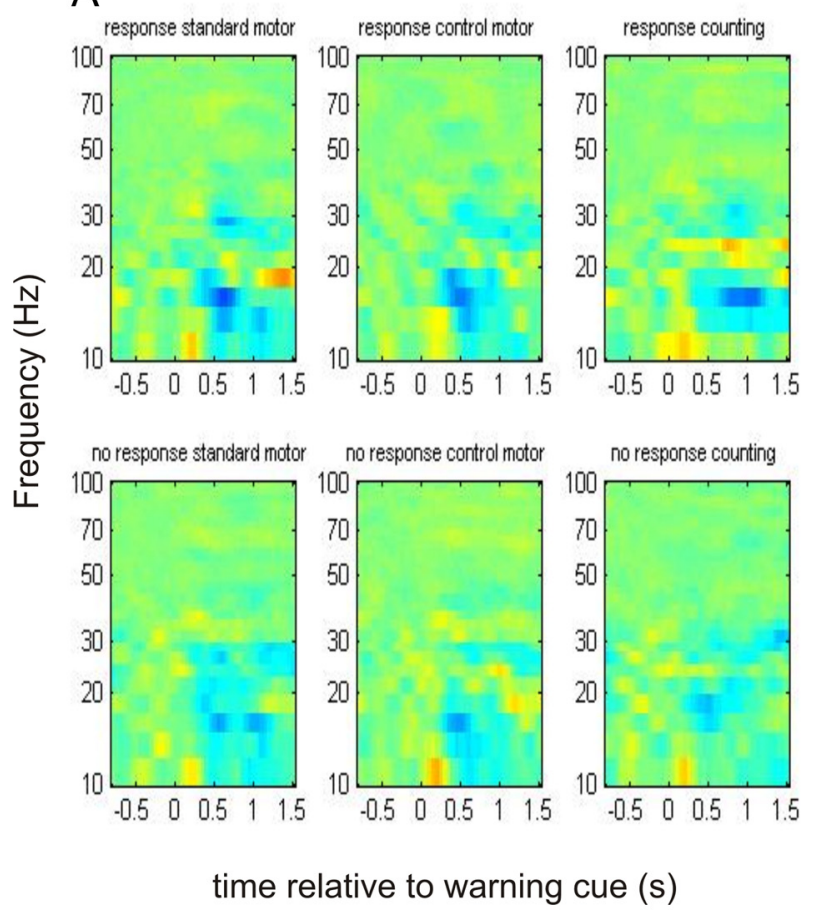

B

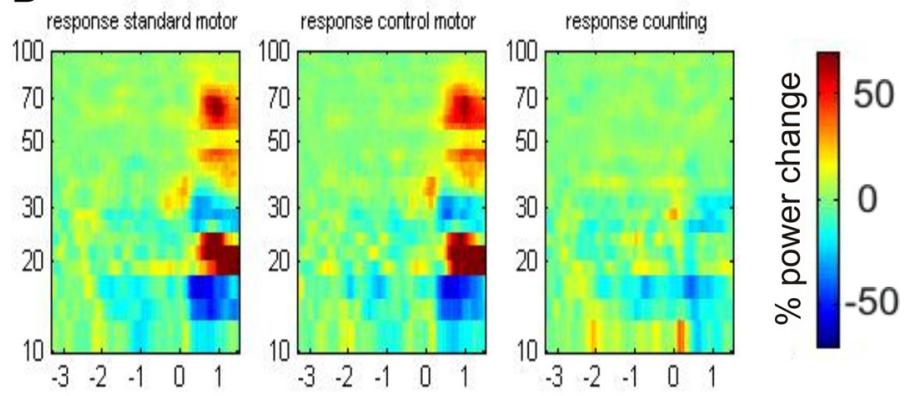

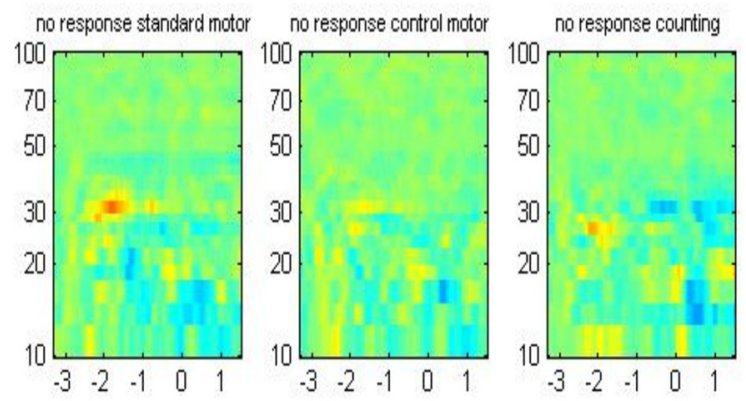

time relative to imperative cue (s)

Figure 2. $\quad \boldsymbol{A}, \boldsymbol{B}$, Time-frequency plots (off levodopa), averaged across all subjects, of the induced responses aligned to the onset of the warning $(\boldsymbol{A})$ and imperative cues ( $\boldsymbol{B})$. For the plots aligned to the onset of the warning cue in $A$, the time window extends to a maximum of $1.5 \mathrm{~s}$, to prevent contamination from any motor responses which could occur after this time period. Power in $\boldsymbol{A}$ was calculated relative to a $0.8 \mathrm{~s}$ period before the appearance of the warning cue. Power in $\boldsymbol{B}$ was calculated relative to a baseline period between $-3.3 \mathrm{~s}$ and $-2.5 \mathrm{~s}$. Note that frequency is plotted on logarithmic axes. Warm colors indicate an increase in power at the respective frequencies, with cooler colors indicating a reduction in power (see color bar). In each panel, the spectra are plotted for the "response" and "no response" conditions of the three different blocks: standard motor, control motor, and non-motor counting task. The main feature to note in $\boldsymbol{B}$ is that the beta band power decrease before the imperative cue appears to be independent of the nature of the "response" warning target. The latter was assessed further statistically using a baseline for normalization of $0.8 \mathrm{~s}$ period before the appearance of the warning cue in Figures 4 and 5 .

counted wrongly in one block, but correctly in the remaining block Off medication. On medication, one patient counted wrongly in one block. Blocks of the counting task were analyzed regardless of the presence of errors in the counted score ( 48 trials in each subject per drug state). There were no erroneous movements made in the counting task.

Recordings. Patients were studied $5 \pm 1 \mathrm{~d}$ postoperatively, in the interval between electrode implantation and subsequent connection to a subcutaneous stimulator. Nine patients were first assessed after overnight withdrawal of antiparkinsonian medication, then $73 \pm 18 \mathrm{~min}$ after a supramaximal dose of levodopa (usual morning dose or $200 \mathrm{mg}$-equivalent levodopa, whichever was higher). One further patient was first recorded On medication and then Off medication on two consecutive days.

LFPs and analog signals related to the cues were recorded through a Biopotential Analyzer Diana (Sechenov Institute of Evolutionary Physiology and Biochemistry, St Petersburg, Russia). LFP activity was recorded monopolarly from the four contacts of each of the 19 deep brain stimulation (DBS) electrodes targeting the STN, referenced to linked earlobes, amplified, filtered $(0.5-300 \mathrm{~Hz})$ and sampled at a common rate of $1200 \mathrm{~Hz}$. Signals were monitored online using software written in our laboratory.

Spectral analysis. Signals were exported for off-line processing in Spike 2 V6 software (Cambridge Electronic Design). So as to minimize volume conduction, we subtracted the signal of the adjacent dorsal electrode contact from each contact to give three bipolar channels per subthalamic electrode (contacts 01,12 , and 23, in which contact pair 01 was the most caudal). We then removed any DC offset by filtering with a time constant of $1 \mathrm{~s}$. The resulting data were exported to MATLAB (MathWorks) for subsequent analysis using custom scripts and the FieldTrip (http:// www.ru.nl/neuroimaging/fieldtrip/) and SPM8 (http://www.fil.ion.ucl. ac.uk/spm/) toolboxes (Litvak et al., 2011; Oostenveld et al., 2011). The data were first epoched into trials, excluding trials with errors, and the average signal over all correct trials was removed from each individual trial to generate the induced response. Spectral analysis was performed for each bipolar electrode pair for all subjects using the multi-taper method (Thomson, 1982). The resulting spectra were subsequently averaged. In our main analysis we averaged across all the contact pairs in a given electrode so as to avoid selection bias. However, because beta oscillations are focally generated in the STN (Kühn et al., 2005; Chen et al., 2006), and not all contacts could be expected to lie in this nucleus, this procedure may underestimate the scale of effects. Therefore, in a subsidiary analysis, we also averaged data across the most reactive contact pair from each electrode, so as to have a better idea of the quantitative effects of our experimental factors and so as to facilitate comparison with other studies that have taken this approach. The most reactive contact pair was defined as the contact pair from each electrode showing the greatest beta desynchronization in the 'response' case $($ STN $01 \times 6$, STN $12 \times 10$, STN $23 \times 3)$.

Spectral analysis was performed for each trial of each of the three conditions, on and off medication for each subject. We separately analyzed the spectra locked to the warning cue and to the target imperative cue between specified time periods (shown in individual figures), in overlapping windows of $400 \mathrm{~ms}$ (shifted by $50 \mathrm{~ms}$ ). The frequency resolution was set to the inverse of the time window $(2.5 \mathrm{~Hz})$ for all frequencies.

The trial data were averaged (regardless of the body part highlighted by the imperative cue) for each subject for statistical analysis across the different conditions ( 6 in total-comprising 3 blocks on and 3 off medication). All analyzed percentage change time-frequency responses were obtained by normalizing to the baseline $(-0.8$ to $-0 \mathrm{~s})$ before the warning cue. Note, however, that the grand averages of responses realigned to warning and imperative cues in Figures 2 and 3 had different baseline periods for the purposes of visualization. This was necessary given that we were averaging across trials with variable intervals between cues.

Statistics. Given that our a priori hypothesis specifically involved searching for differences in the beta frequency band $(15-30 \mathrm{~Hz})$, we averaged the spectral data over this band by computing a multi-taper 

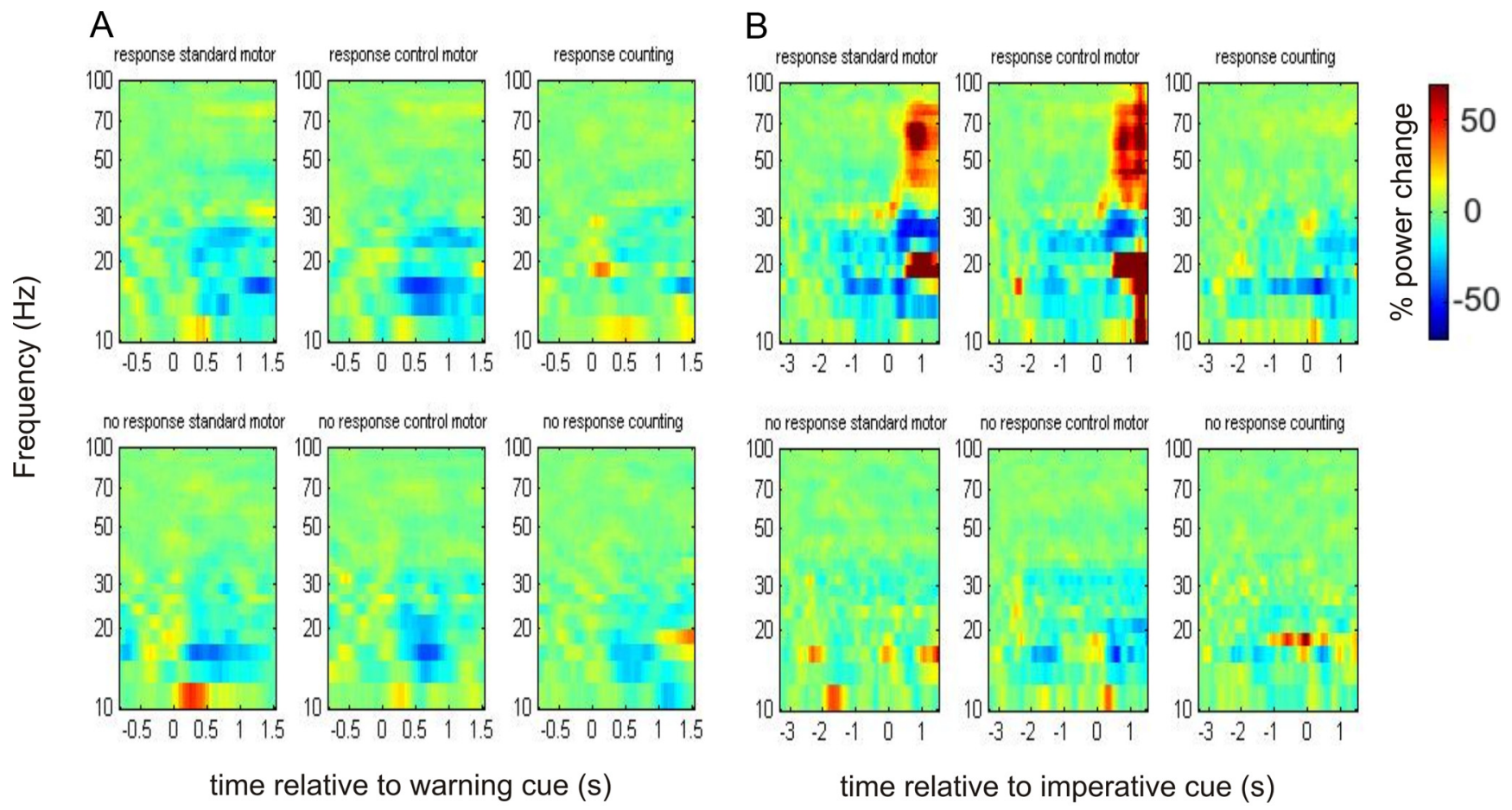

Figure 3. Time-frequency plots as per Figure 2, following the administration of levodopa. Induced responses are aligned to the onset of the warning $(\boldsymbol{A})$ and imperative $(\boldsymbol{B})$ cues. There is a clearer pattern of beta desynchronization in the response trials before the onset of the imperative cue.

spectral estimate with central frequency of $22.5 \mathrm{~Hz}$ and frequency resolution of $7.5 \mathrm{~Hz}$. This is a more efficient way of estimating beta power than averaging over pre-computed time-frequency images with higher frequency resolution (Mitra and Pesaran, 1999). The averaged beta time series for each condition and electrode were converted into 1D images (Neuroimaging Informatics Technology Initiative format) for statistical analysis in SPM8. Here, we were able to correct for multiple comparisons in time using random field theory (Kilner et al., 2005).

Using this approach we analyzed the period between the warning and imperative cues independently for the motor and non-motor tasks using a repeated-measures ANOVA for the 10 subjects. We also included subject as a covariate in the design to account for the fact that the time series from each pair of right and left subthalamic nuclei were from the same subject. Additionally we included a side covariate for each subject to account for potential differences between recordings from the right and left subthalamic nuclei. For the motor task, the factors were Block (Motor Task vs Control Motor Task), Cue ("response" vs "no response"), and Dopaminergic state (ON vs OFF). For the non-motor task, the factors were Cue and Dopaminergic state only. We did not analyze signals after presentation of the imperative cue.

Using this repeated measures design in SPM, we specified $t$ contrasts to test for times at which significant differences occurred using paired $t$ tests. Using the $t$ contrasts in SPM, we were able to test for the direction of significance; for example, taking the main effect of cue, we could test whether "response" produced significantly less desynchronization than "no response" or vice versa. All the reported findings are significant with familywise error (FWE) correction at the peak level $(p<0.05)$. In addition, we tested the statistical significance of changes in each time series of beta power relative to baseline by performing serial one-sample $t$ tests in time and correcting for multiple comparisons in SPM8 using random field theory. In the latter case we made our procedure even more conservative by considering only those changes from baseline that were significant over three or more contiguous time points ( $\geq 150 \mathrm{~ms}$ ).

In a separate analysis, we performed the same statistics as described above looking for significant differences in the gamma band $(60-90 \mathrm{~Hz})$ across conditions for all subjects. Finally, to confirm the focal origin of the LFP beta activity, we defined the contact pair showing the maximum beta power in each electrode as $100 \%$ and calculated the mean relative beta power at the remaining contact pairs. The latter were averaged across electrodes, and the mean relative power and its SE are presented.

\section{Results}

Time-frequency plots of the induced responses in the 3 different tasks to "response" and "no response" warning cues OFF medication are presented in Figure 2. To the left side of the figure, data are averaged with respect to the onset of the warning cue (given as time 0 ), and again presented relative to a prestimulus baseline of $0.8 \mathrm{~s}$. In the left-hand images the time extends only to $1.5 \mathrm{~s}$ after the onset of the warning to prevent contamination from motor responses, which could occur after a minimum foreperiod of $1.5 \mathrm{~s}$. To the right side of the figure, the data are averaged with respect to the appearance of the imperative cue (given as time 0 ), and presented relative to a baseline period between $-3.3 \mathrm{~s}$ and -2.5 s. Figure 3 shows the corresponding plots ON medication.

Beta desynchronizations were greater following the "response" than the "no response" warning cues when ON (Fig. 3) compared with OFF (Fig. 2) medication, and in the motor tasks this appeared to be independent of the nature of the "response" warning target. These effects were also seen in the one subject who was first tested $\mathrm{ON}$ medication, i.e., in the reverse order to the other subjects, suggesting that any effects of dopaminergic state were unlikely to have been confounded by the order of task performance.

The effects were confirmed at the group level in an ANOVA with factors Target (circle vs square/triangle), Warning Cue ("response" vs "no response"), and Medication (ON vs OFF) in the motor task, where there was no main effect or interaction with target. Accordingly, we amalgamated data from the two motor tasks, in a $2 \times 2$ ANOVA with factors "warning Cue ("response" vs "no response") and medication (ON vs OFF) to test for the main effects and interaction of warning cue and medication. Figure 4 (top) shows the averaged beta power time series across all subjects in the motor tasks $\mathrm{ON}$ and OFF medication aligned to 


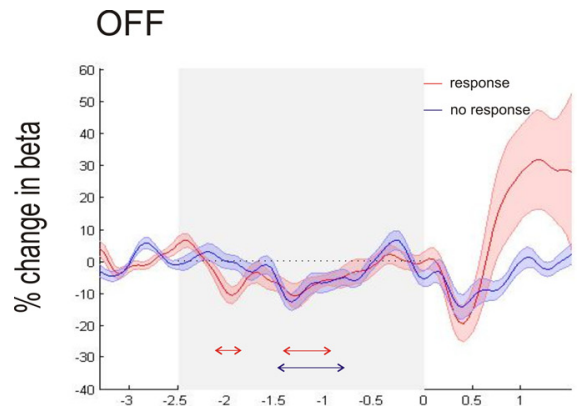

ON
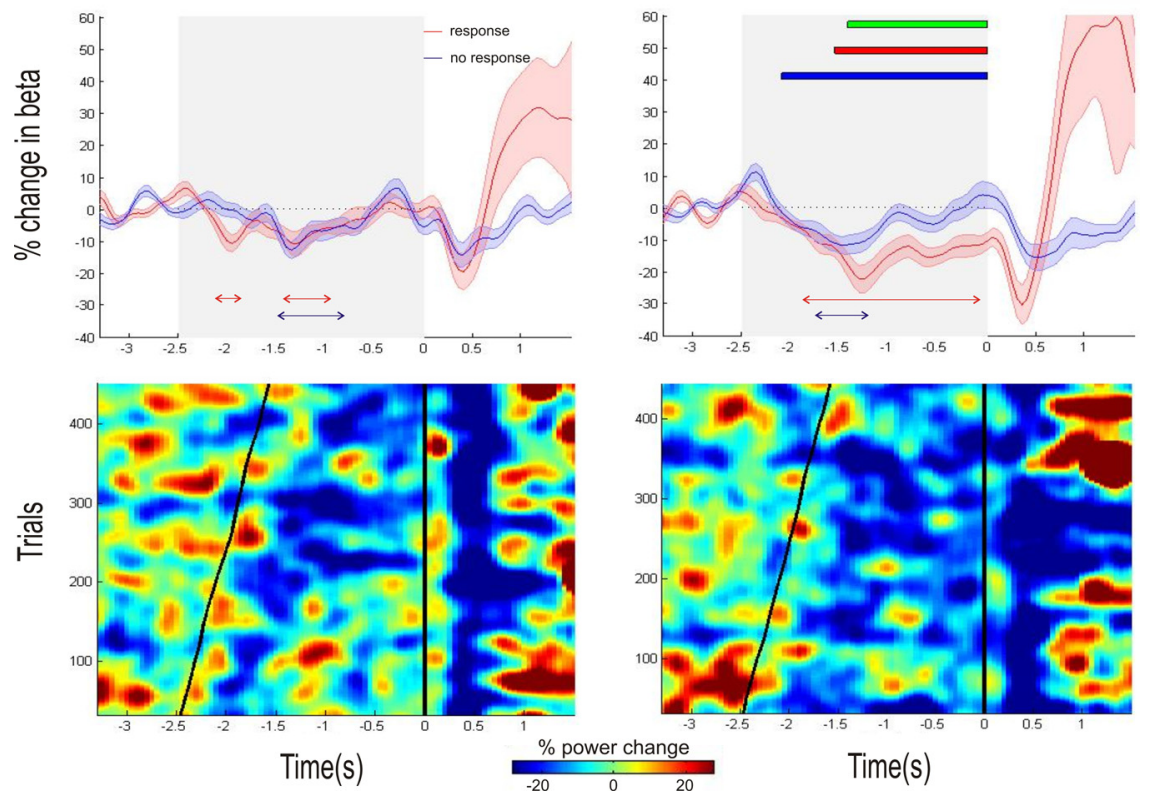

Figure 4. Top, Mean time series of the percentage change in beta power (shaded areas indicating the SEM) relative to a $0.8 \mathrm{~s}$ baseline in the motor task for the response and no response conditions. The time series are plotted separately for the OFF and ON medication states, with time 0 representing the onset of the imperative cue. The gray shaded area indicates the time between the maximal foreperiod $(-2.5 \mathrm{~s})$ and the onset of the imperative cue. Below the time series, the red and blue arrowed lines indicate times at which there was a significant reduction of beta power, occurring consistently over a period of at least $150 \mathrm{~ms}$ (FWE corrected, $p<0.05$ ) for the response and no response conditions, respectively. Also plotted in the figure to the right are the times of: (1) significant main effect of medication (red solid bar), (2) significant main effect of cue (blue solid bar), and (3) significant interaction between medication and cue (green solid bar). Bottom, Rasters of trial beta power for all the motor response trials of all subjects. Cool colors indicate a reduction in beta power, with warmer colors indicating the opposite (see color bar). The trials are ordered by foreperiod, with the onset of the warning and imperative cues corresponding to the diagonal and vertical black lines. The noisy individual trial data were smoothed by convolution with a Gaussian before plotting. Separate images are plotted for the $\mathrm{OFF}$ and $\mathrm{ON}$ medication states. There is greater and more prolonged beta desynchronization $\mathrm{ON}$ medication, with maximal desynchronization occurring after the onset of the warning cue.

\section{OFF}

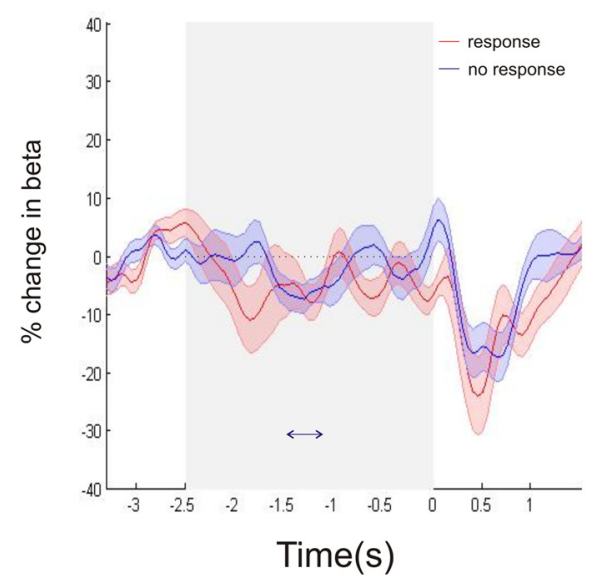

ON

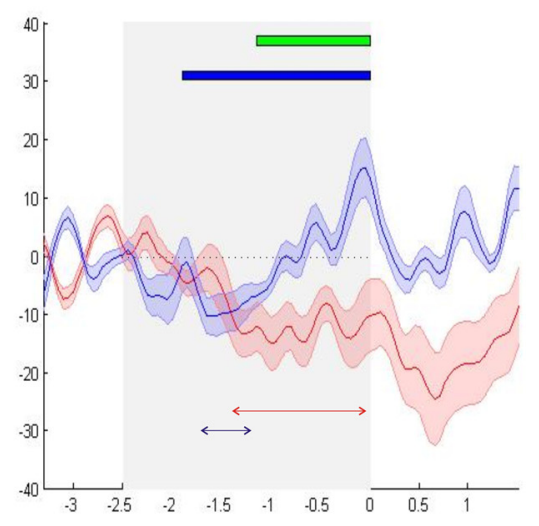

I Ime(S)
Figure 5. Mean time series of the percentage change in beta power (blue and red shaded regions indicating SEM) relative to a $0.8 \mathrm{~s}$ baseline in the counting task for the response and no response conditions. The time series are plotted separately for the $0 \mathrm{FF}$ and $0 \mathrm{~N}$ medication states, aligned to the onset of the imperative cue. The gray shaded area indicates the time between the maximal foreperiod $(-2.5 \mathrm{~s})$ and the onset of the imperative cue. Below the time series, the blue arrowed line indicates times at which there was a significant increase in beta power, occurring consistently over a period of at least $150 \mathrm{~ms}$ (FWE corrected, $p<$ $0.05)$ for the no response condition. Below the time series the red and blue arrowed lines indicate times at which there was a significant reduction in beta power occurring consistently over a period of at least $150 \mathrm{~ms}$ (FWE corrected, $p<0.05$ ) for the response and no response conditions, respectively. Also plotted in the figure to the right are the times of: (1) significant main effect of cue (blue solid bar) and (2) significant interaction between medication and cue (green solid bar). the onset of the imperative cue. On the right side of the top panel the times of significance of the main effects of cue and medication and the interaction of cue and medication are indicated. (The time points of peak $t$ scores for each main effect and interaction had the following statistics: main effect of cue- $t_{(5.93)}, \mathrm{df}=209$, $p<0.001$; main effect of medication$t_{(4.17)}, \mathrm{df}=209, p<0.001$; cue and medication interaction- $t_{(4.19)}, \mathrm{df}=209, p<$ 0.001.) Beta desynchronization was greater on medication than off medication and greater with "response" warning cues compared with "no response" warning cues. There was also an interaction whereby the difference in beta desynchronization induced by "response" and "no response" warning cues $\mathrm{ON}$ medication exceeded that OFF medication.

The same analysis is shown for the non-motor counting task in Figure 5. Here there was no main effect for medication, but, as with the motor task, there was a main effect of warning cue and an interaction between medication and cue (the time points of peak $t$ scores for each main effect and interaction had the following statistics: main effect of cue $-t_{(4.97)}$, $\mathrm{df}=$ $209, p<0.001$; cue and medication interaction- $\left.t_{(4.23)}, \mathrm{df}=209, p<0.001\right)$.

Note that in the "response" and "no response" conditions for both tasks on dopamine, there appears to be a gradual beta desynchronization over the first $\sim 1.0$ s. This is an inevitable product of the variable foreperiod and comes about through the temporal smearing of the relatively sharp beta desynchronizations following the warning cue. The temporal smearing is induced by the variable inter-cue interval, as can be seen in the rasters of trial beta activity presented in the bottom of Figure 4. More importantly, the beta desynchronization is subsequently maintained following the "response" warning cue but is not maintained following the "no response" warning cue (Fig. 4, top and bottom). Interestingly, for the counting task ON medication, there was a statistically significant increase in beta power for the "no response" condition just before the imperative cue (Fig. 5). There was only a trend toward this effect in the motor task ON medication (Fig. 4), when this task was considered in isolation. To further explore this specific feature, we contrasted the mean beta power for each electrode in the final $200 \mathrm{~ms}$ before the imperative cue in "no response" trials with the mean beta power in the $200 \mathrm{~ms}$ before presentation of the warning "no response" cue in corresponding trials in 
a repeated-measures ANOVA. We considered Period (pre-warning vs preimperative), Task Type (standard motor vs control motor vs non-motor counting), and Medication (ON vs OFF) as our main effects, and specifically sought an interaction between period and medication. This was confirmed, so that mean beta power before the imperative cue exceeded that before the corresponding warning cue, but only ON medication $\left(F_{(2,216)}=\right.$ $4.54 p=0.0118)$. There was also a significant interaction among the three factors period, medication, and task type $\left(F_{(2,216)}=\right.$ $3.91, p=0.0214)$, which arose because the effect of medication on the period before the imperative cue was greatest in the nonmotor counting task.

In further analysis, we recomputed beta time series, after including only the "most reactive" contact pair for each electrode. The results are illustrated in Figure 6 in which, as before, times of significance of the main effects of cue, medication, and their interaction are indicated. The figure shares the key features of Figures 4 and 5. In addition, and in line with the interaction between period and medication in the ANOVA performed above, it is now clear that there is a statistically significant increase in beta power for both the motor and counting tasks before the onset of the imperative cue in "no response" trials made $\mathrm{ON}$ medication. This was evident even when activity at the most reactive contact pair was considered in isolation for each task.

The foreperiod effects of our experimental factors were frequency selective (Figs. 2, 3). In a separate analysis of the gamma frequency band, we did not find any significant main effects or interactions for the motor or counting paradigms. Finally, the LFP power in the beta band dropped by a mean of $54.2 \pm 9.9 \%$ when the contact pair with the highest baseline beta power was contrasted with the remaining contact pairs of each electrode in the OFF medication state. In 12 of 19 cases, the contact with the greatest baseline beta power was that with the greatest beta desynchronization in the "response" case.

\section{Discussion}

We recorded STN LFPs to warning cues that indicated whether a response would or would not be required in an upcoming motor or cognitive task. To maximally dissociate processing related to the saliency of the warning cue from task-specific processing, we systematically varied both the timing of the imperative cue after the warning cue and the specific nature of the task to be performed. The core finding was that changes in beta power in the LFP depended on the behavioral relevance of the warning cue. The paradigm ruled out conflation of this with motor-related processing, while a control in which the nature of the target and non-target warning cues was reversed confirmed that power changes were not simply related to the sensory processing of the specific form of the target. Warning cues that pointed to the need for a subsequent response were followed by beta power suppression, whereas those that signaled that no response would be nec-
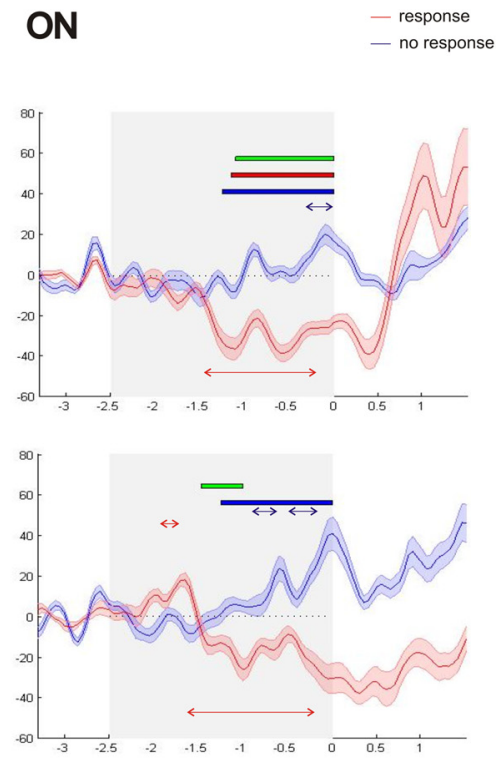

Time(s)

Figure 6. Beta time series are plotted $O N$ and OFF medication for the most reactive contact pairs across subjects in the motor (top) and counting (bottom) tasks. The most reactive contact pair was defined as the contact pair from each electrode showing the

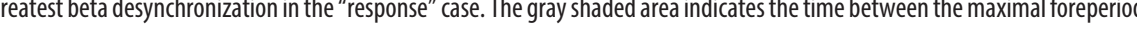

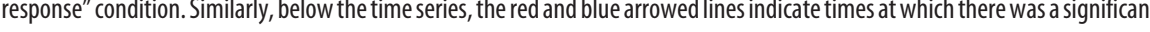
of: (1) significant main effect of medication (red solid bar), (2) significant main effect of cue (blue solid bar), and (3) significant interaction between medication and cue (green solid bar).

essary were followed by delayed beta power increases, which preceded the imperative cue. Beta power changes following warning cues signaling the need or not for a subsequent response occurred regardless of whether the necessary response was motor or cognitive, as in the counting task. This provides further evidence that the foreperiod responses were not confounded by motor processing and supports a wider role for beta power modulation in executive function at both subcortical and cortical levels (Engel and Fries, 2010).

This homology between the beta reactivity in the foreperiod of both motor and cognitive tasks extended to a similar dependence on dopamine. Beta reactivity to warning cues was potentiated in the dopamine replenished on medication state, as evinced by an interaction between cue and drug in both tasks. The dopamine dependency of beta reactivity in the motor task is consistent with current theories of the nature of oscillatory activity in this frequency band (Jenkinson and Brown, 2011), and there is good evidence that the prevailing level of beta activity in the motor regions of the basal ganglia can be altered by drugs that manipulate dopamine and its receptors, both in patients with PD (Hammond et al., 2007) and those without PD (Kühn et al., 2008). The dopamine dependency of beta reactivity in the cognitive task is perhaps more unexpected, but would be in keeping with observations of bradyphrenia and cognitive inflexibility treatable with dopamine in some patients with PD (Rogers et al., 1987; Lees, 1994; Zgaljardic et al., 2003).

What precisely do the foreperiod power changes in the beta band signal? As highlighted above, they depended on the salience of the warning cue, specifically if it predicted or not the possible need for a subsequent response. But they were unlikely to merely 
relate to saliency as the power differences were more-or-less sustained up to the point of presentation of the imperative cue, and even the no-response cues could be considered to have some behavioral saliency. This makes it more likely that the foreperiod power changes in the beta band relate to anticipation of the motor or cognitive response (and its attendant processing) engendered by the saliency of the cue, rather than cue saliency per se. Such an anticipatory process could subserve predictive resourcing of the prospective action and economy of resourcing in the absence of the need for action. This possibility would be in accord with recent theories of the functional role of oscillatory activity in the beta band (Jenkinson and Brown, 2011). The latter posit that beta activity provides a dynamic indicator of the likelihood that a new action will need to be actuated, with the level of beta being inversely proportional to the likelihood that a new action will need to be processed and performed. Alternatively, the sustained beta power changes might relate to working memory processes enlisted by the warning cue. However, the latter seems unlikely as the working memory load of both "response" and "no response" warning cues would be similar, and yet differential power changes occurred in opposite directions.

The dopamine-dependent increase in beta power before the imperative cue in "no response" trials merits further comment. The increase presumably reflects a ramping of beta activity with temporal expectancy of the imperative cue (Androulidakis et al., 2007), and is similar to that reported after nogo imperative cues (Kühn et al., 2004). In the latter case, the beta power increase was interpreted as evidence of an active inhibitory process, consistent with current views that afford the STN a critical role in motor inhibition (Frank et al., 2007; Ray et al., 2009, 2012; Forstmann et al., 2010; Cavanagh et al., 2011). By analogy, the power increase before the imperative cue in the current paradigm could relate to the inhibition of a prepotent response to the imperative cue. However, "response" and "no response" trials occurred with equal frequency and, more importantly, the nature of the response to be inhibited was unknown at this point in time. We would rather view the beta power increase in the same context as the prospective (anticipatory) beta power decrease occurring in "response" trials. Thus, the beta power increase at the end of the foreperiod of "no response" trials might reflect the predictive withdrawal of resources in the absence of the need for action, which, inevitably, also reduces the likelihood of any erroneous prepotent response.

In summary, the pattern of modulation of STN beta activity reported here is consistent with an active process that prospectively sets the state of readiness for executive processing (be it primarily motor or cognitive), based on the salience of incoming cues. The alternative, that beta suppression could be an epiphenomenon of the shift in neuronal patterning that underscores executive processing, seems unlikely as our paradigm dissociated beta power modulation in the foreperiod from any motor-related processing. The latter only supervened following the imperative cue, as evidenced by the change in gamma activity at this point, but not before. Even so, the percentage changes in beta power during the foreperiod were of the same order of magnitude as those reported in studies in which taskspecific processing has not been excluded from this period (Williams et al., 2003; Kühn et al., 2004, 2006).

Note that we analyzed induced power changes in this study and so explored separate processes to the averaged evoked time domain signals previously reported in this paradigm (Sauleau et al., 2009). Nevertheless, it is interesting to note that the behavior of evoked responses was similar, with dependencies on cue salience and levodopa. Although this evoked response study was helpful in em- phasizing the role of the STN in registering behavioral salience, it did not set this in the broader context of the function of beta activity.

We studied patients, so that inferences with regard to normal functioning must be circumspect (Williams et al., 2002). That said, the predictive modulation of basal ganglia beta activity brought on by salient cues tends to parallel the behavior of beta activity in the cerebral cortex of healthy subjects (Androulidakis et al., 2007; Donner et al., 2009; van Wijk et al., 2009; Tzagarakis et al., 2010; van Ede et al., 2010; Gould et al., 2011). Of particular note, reduction in the power of cortical beta-band activity during motor preparation scales with the directional uncertainty of forthcoming movement, and this in turn correlates with the change of latency with response uncertainty (Tzagarakis et al., 2010). Other limitations are the presumptive localization of electrode contacts in the STN (Williams et al., 2002; Fogelson et al., 2006) and the possibility that power changes picked up at the bipolar contacts of the DBS electrode are not really focally generated. Against the latter, we found a steep gradient in LFP power in the beta band across different bipolar contact pairs consistent with a local generator (Kühn et al., 2004, 2006), although the strongest argument in favor of the local generation of beta activity in the LFP is the demonstration that the discharge of neurons in the STN tends to be locked to it (Levy et al., 2002; Kühn et al., 2005). A more specific limitation in the current study is that the paradigm was not designed to evaluate whether beta activity was modulated by the temporal expectancy of the imperative cue. This would have helped support the hypothesis that beta modulation prospectively sets the state of readiness for executive processing, but the paradigm did not include a separate training phase where subjects were exposed to multiple trials, allowing them to learn the distribution of foreperiod durations, and we did not give feedback to facilitate learning.

In conclusion, we have provided evidence that the modulation of beta activity in the STN is not simply an epiphenomenon of the reconfiguration of neural interactions in favor of synchronization at higher frequency during task-related processing, but is the consequence of an active process that may prospectively set the state of readiness for executive processing according to the salience of incoming cues. This reactivity in beta band oscillations is impaired when dopamine is deficient.

\section{References}

Alegre M, Alonso-Frech F, Rodríguez-Oroz MC, Guridi J, Zamarbide I, Valencia M, Manrique M, Obeso JA, Artieda J (2005) Movement-related changes in oscillatory activity in the human subthalamic nucleus: ipsilateral vs contralateral movements. Eur J Neurosci 22:2315-2324.

Amirnovin R, Williams ZM, Cosgrove GR, Eskandar EN (2004) Visually guided movements suppress subthalamic oscillations in Parkinson's disease patients. J Neurosci 24:11302-11306.

Androulidakis AG, Doyle LM, Yarrow K, Litvak V, Gilbertson TP, Brown P (2007) Anticipatory changes in beta synchrony in the human corticospinal system and associated improvements in task performance. Eur J Neurosci 25:3758-3765.

Androulidakis AG, Brücke C, Kempf F, Kupsch A, Aziz T, Ashkan K, Kühn AA, Brown P (2008) Amplitude modulation of oscillatory activity in the subthalamic nucleus during movement. Eur J Neurosci 27:1277-1284.

Cavanagh JF, Wiecki TV, Cohen MX, Figueroa CM, Samanta J, Sherman SJ, Frank MJ (2011) Subthalamic nucleus stimulation reverses mediofrontal influence over decision threshold. Nat Neurosci 14:1462-1467.

Chen CC, Pogosyan A, Zrinzo LU, Tisch S, Limousin P, Ashkan K, Yousry T, Hariz MI, Brown P (2006) Intra-operative recordings of local field potentials can help localize the subthalamic nucleus in Parkinson's disease surgery. Exp Neurol 198:214-221.

Chen CC, Lin WY, Chan HL, Hsu YT, Tu PH, Lee ST, Chiou SM, Tsai CH, Lu CS, Brown P (2011) Stimulation of the subthalamic region at $20 \mathrm{~Hz}$ slows the development of grip force in Parkinson's disease. Exp Neurol 231:91-96.

Courtemanche R, Fujii N, Graybiel AM (2003) Synchronous, focally modulated beta-band oscillations characterize local field potential activity in the striatum of awake behaving monkeys. J Neurosci 23:11741-11752. 
Devos D, Szurhaj W, Reyns N, Labyt E, Houdayer E, Bourriez JL, Cassim F, Krystkowiak P, Blond S, Destée A, Derambure P, Defebvre L (2006) Predominance of the contralateral movement-related activity in the subthalamo-cortical loop. Clin Neurophysiol 117:2315-2327.

Donner TH, Siegel M, Fries P, Engel AK (2009) Buildup of choicepredictive activity in human motor cortex during perceptual decision making. Curr Biol 19:1581-1585.

Doyle LMF, Kühn AA, Hariz M, Kupsch A, Schneider GH, Brown P (2005) Levodopa-induced modulation of subthalamic beta oscillations during self-paced movements in patients with Parkinson's disease. Eur J Neurosci 21:1403-1412.

Engel AK, Fries P (2010) Beta-band oscillations-signalling the status quo? Curr Opin Neurobiol 20:156-165.

Foffani G, Priori A, Egidi M, Rampini P, Tamma F, Caputo E, Moxon KA, Cerutti S, Barbieri S (2003) 300-Hz subthalamic oscillations in Parkinson's disease. Brain 126:2153-2163.

Fogelson N, Williams D, Tijssen M, van Bruggen G, Speelman H, Brown P (2006) Different functional loops between cerebral cortex and the subthalmic area in Parkinson's disease. Cereb Cortex 16:64-75.

Forstmann BU, Anwander A, Schäfer A, Neumann J, Brown S, Wagenmakers EJ, Bogacz R, Turner R (2010) Cortico-striatal connections predict control over speed and accuracy in perceptual decision making. Proc Natl Acad Sci U S A 107:15916-15920.

Frank MJ, Samanta J, Moustafa AA, Sherman SJ (2007) Hold your horses: impulsivity, deep brain stimulation, and medication in parkinsonism. Science 318:1309-1312.

Gould IC, Rushworth MF, Nobre AC (2011) Indexing the graded allocation of visuospatial attention using anticipatory alpha oscillations. J Neurophysiol 105:1318-1326.

Hammond C, Bergman H, Brown P (2007) Pathological synchronization in Parkinson's disease: networks, models and treatments. Trends Neurosci 30:357-364.

Jenkinson N, Brown P (2011) New insights into the relationship between dopamine, beta oscillations and motor function. Trends Neurosci 34:611-618.

Kane A, Hutchison WD, Hodaie M, Lozano AM, Dostrovsky JO (2009) Dopamine-dependent high-frequency oscillatory activity in thalamus and subthalamic nucleus of patients with Parkinson's disease. Neuroreport 20:1549-1553.

Kempf F, Kühn AA, Kupsch A, Brücke C, Weise L, Schneider GH, Brown P (2007) Premovement activities in the subthalamic area of patients with Parkinson's disease and their dependence on task. Eur J Neurosci 25:3137-3145.

Kilner JM, Kiebel SJ, Friston KJ (2005) Applications of random field theory to electrophysiology. Neurosci Lett 374:174-178.

Kühn AA, Williams D, Kupsch A, Limousin P, Hariz M, Schneider GH, Yarrow K, Brown P (2004) Event-related beta desynchronization in human subthalamic nucleus correlates with motor performance. Brain 127:735-746.

Kühn AA, Trottenberg T, Kivi A, Kupsch A, Schneider GH, Brown P (2005) The relationship between local field potential and neuronal discharge in the subthalamic nucleus of patients with Parkinson's disease. Exp Neurol 194:212-220.

Kühn AA, Doyle L, Pogosyan A, Yarrow K, Kupsch A, Schneider GH, Hariz MI, Trottenberg T, Brown P (2006) Modulation of beta oscillations in the subthalamic area during motor imagery in Parkinson's disease. Brain 129:695-706.

Kühn AA, Brücke C, Schneider GH, Trottenberg T, Kivi A, Kupsch A, Capelle $\mathrm{HH}$, Krauss JK, Brown P (2008) Increased beta activity in dystonia patients after drug-induced dopamine deficiency. Exp Neurol 214:140-143.

Kühn AA, Tsui A, Aziz T, Ray N, Brücke C, Kupsch A, Schneider GH, Brown P (2009) Pathological synchronisation in the subthalamic nucleus of patients with Parkinson's disease relates to both bradykinesia and rigidity. Exp Neurol 215:380-387.

Lees AJ (1994) The concept of bradyphrenia. Rev Neurol 150:823-826.

Levy R, Hutchinson WD, Lozano AM, Dostrovsky JO (2002) Synchronized neuronal discharge in the basal ganglia of parkinsonian patients is limited to oscillatory activity. J Neurosci 22:2855-2861.

Litvak V, Mattout J, Kiebel S, Phillips C, Henson R, Kilner J, Barnes G, Oostenveld R, Daunizeau J, Flandin G, Penny W, Friston K (2011) EEG and MEG data analysis in SPM8. Comput Intell Neurosci 2011:852961.
López-Azcárate J, Tainta M, Rodríguez-Oroz MC, Valencia M, González R, Guridi J, Iriarte J, Obeso JA, Artieda J, Alegre M (2010) Coupling between beta and high-frequency activity in the human subthalamic nucleus may be a pathophysiological mechanism in Parkinson's disease. J Neurosci 30:6667-6677.

Mitra PP, Pesaran B (1999) Analysis of dynamic brain imaging data. Biophys J 76:691-708.

Oostenveld R, Fries P, Maris E, Schoffelen JM (2011) FieldTrip: Open source software for advanced analysis of MEG, EEG, and invasive electrophysiological data. Comput Intell Neurosci 2011:156869.

Özkurt TE, Butz M, Homburger M, Elben S, Vesper J, Wojtecki L, Schnitzler A (2011) High frequency oscillations in the subthalamic nucleus: a neurophysiological marker of the motor state in Parkinson's disease. Exp Neurol 229:324-331.

Pogosyan A, Kühn AA, Trottenberg T, Schneider GH, Kupsch A, Brown P (2006) Elevations in local gamma activity are accompanied by changes in the firing rate and information coding capacity of neurons in the region of the subthalamic nucleus in Parkinson's disease. Exp Neurol 202:271-279.

Pogosyan A, Gaynor LD, Eusebio A, Brown P (2009) Boosting cortical activity at Beta-band frequencies slows movement in humans. Curr Biol 19:1637-1641.

Ray NJ, Jenkinson N, Wang S, Holland P, Brittain JS, Joint C, Stein JF, Aziz T (2008) Local field potential beta activity in the subthalamic nucleus of patients with Parkinson's disease is associated with improvements in bradykinesia after dopamine and deep brain stimulation. Exp Neurol 213:108-113.

Ray NJ, Jenkinson N, Brittain J, Holland P, Joint C, Nandi D, Bain PG, Yousif N, Green A, Stein JS, Aziz TZ (2009) The role of the subthalamic nucleus in response inhibition: evidence from deep brain stimulation for Parkinson's disease. Neuropsychologica 47:2828-2834.

Ray NJ, Brittain JS, Holland P, Joundi RA, Stein JF, Aziz TZ, Jenkinson N (2012) The role of the subthalamic nucleus in response inhibition: evidence from local field potential recordings in the human subthalamic nucleus. Neuroimage 60:271-278.

Rogers D, Lees AJ, Smith E, Trimble M, Stern GM (1987) Bradyphrenia in Parkinson's disease and psychomotor retardation in depressive illness. An experimental study. Brain 110:761-776.

Sauleau P, Eusebio A, Thevathasan W, Yarrow K, Pogosyan A, Zrinzo L, Ashkan K, Aziz T, Vandenberghe W, Nuttin B, Brown P (2009) Involvement of the subthalamic nucleus in engagement with behaviourally relevant stimuli. Eur J Neurosci 29:931-942.

Thomson DJ (1982) Spectrum estimation and harmonic analysis. Proc IEEE 70:1055-1096.

Tzagarakis C, Ince NF, Leuthold AC, Pellizzer G (2010) Beta-band activity during motor planning reflects response uncertainty. J Neurosci 30:11270-11277.

van Ede F, Jensen O, Maris E (2010) Tactile expectation modulates prestimulus beta-band oscillations in human sensorimotor cortex. Neuroimage 51:867-876.

van Wijk BC, Daffertshofer A, Roach N, Praamstra P (2009) A role of beta oscillatory synchrony in biasing response competition? Cereb Cortex 19:1294-1302.

Williams D, Tijssen M, Van Bruggen G, Bosch A, Insola A, Di Lazzaro V, Mazzone P, Oliviero A, Quartarone A, Speelman H, Brown P (2002) Dopamine-dependent changes in the functional connectivity between basal ganglia and cerebral cortex in humans. Brain 125:1558-1569.

Williams D, Kühn A, Kupsch A, Tijssen M, van Bruggen G, Speelman H, Hotton G, Yarrow K, Brown P (2003) Behavioural cues are associated with modulations of synchronous oscillations in the human subthalamic nucleus. Brain 126:1975-1985.

Williams D, Kühn A, Kupsch A, Tijssen M, van Bruggen G, Speelman H, Hotton G, Loukas C, Brown P (2005) The relationship between oscillatory activity and motor reaction time in the parkinsonian subthalamic nucleus. Eur J Neurosci 21:249-258.

Zaidel A, Spivak A, Grieb B, Bergman H, Israel Z (2010) Subthalamic span of beta oscillations predicts deep brain stimulation efficacy for patients with Parkinson's disease. Brain 133:2007-2021.

Zgaljardic DJ, Borod JC, Foldi NS, Mattis P (2003) A review of the cognitive and behavioral sequelae of Parkinson's disease: relationship to frontostriatal circuitry. Cogn Behav Neurol 16:193-210. 